\title{
Existence uniqueness of mild solutions for $\psi$-Caputo fractional stochastic evolution equations driven by $\mathrm{fBm}$
}

\author{
Min Yang ${ }^{1 *}$
}

*Correspondence:

yangm58@mail2.sysu.edu.cn

${ }^{1}$ College of Mathematics, Taiyuan

University of Technology, 030024

Taiyuan, China

\section{Springer}

\begin{abstract}
In this paper, we investigate the existence uniqueness of mild solutions for a class of $\psi$-Caputo fractional stochastic evolution equations with varying-time delay driven by $\mathrm{fBm}$, which seems to be the first theoretical result of the $\psi$-Caputo fractional stochastic evolution equations. Alternative conditions to guarantee the existence uniqueness of mild solutions are obtained using fractional calculus, stochastic analysis, fixed point technique, and noncompact measure method. Moreover, an example is presented to illustrate the effectiveness and feasibility of the obtained abstract results.
\end{abstract}

Keywords: $\psi$-Caputo fractional derivative; Stochastic evolution equations; Noncompact measure; Fractional Brownian motion

\section{Introduction}

Fractional differential equations grew to be a popular research topic for their wide applications in engineering, mathematics, physics, bio-engineering, and other applied sciences. Considerable work has been done in this area in recent years, both in theory and applications. Citing all papers and books of this field will be impossible. Therefore, here we only recommend the readers interested in this area refer to [1, 3, 8-12, 16, 21, 24, 27-29, 33-35] for more details on the theory and applications of fractional differential equations.

In particular, Kiryakova [18] proposed a theory of generalized fractional calculus (generalizations of fractional integrals and derivatives) and discussed its applications. We can find generalized fractional integrals and derivatives with specific functions $\psi(t)$ and with weights $w(t)$ in [18] and [25]. To be specific, it can be easily noticed that when $\psi(t)=t, w(t)=1, \psi$-Riemann-Liouville fractional derivative coincides with the classical Riemann-Liouville fractional derivative, $\psi$-Caputo fractional derivative is actually the classical Caputo fractional derivative. When $\psi(t)=\ln t, w(t)=1$, $\psi$-Riemann-Liouville fractional derivative coincides with the Hadamard fractional derivative. On the other hand, Almeida [2] studied some properties of $\psi$-Caputo fractional derivative by considering the Caputo fractional derivative of a function with respect to another function $\psi$. The advantage of this new definition of the fractional derivative is that a higher accuracy

(c) The Author(s) 2021. This article is licensed under a Creative Commons Attribution 4.0 International License, which permits use sharing, adaptation, distribution and reproduction in any medium or format, as long as you give appropriate credit to the original author(s) and the source, provide a link to the Creative Commons licence, and indicate if changes were made. The images or other third party material in this article are included in the article's Creative Commons licence, unless indicated otherwise in a credit line to the material. If material is not included in the article's Creative Commons licence and your intended use is not permitted by statutory regulation or exceeds the permitted use, you will need to obtain permission directly from the copyright holder. To view a copy of this licence, visit http://creativecommons.org/licenses/by/4.0/. 
of the model could be achieved with the choice of a suitable function $\psi$. In addition, by applying Laplace transform and probability density functions, Suechoei and Sa Ngiamsunthorn [26] studied the local and global existence and uniqueness of mild solutions to the following fractional evolution equation of the form:

$$
\left\{\begin{array}{l}
{ }_{0}^{C} D_{\alpha}^{\psi} x(t)=A x(t)+f(t, x(t)), \quad t \in(0, b] \\
x(0)=x_{0}
\end{array}\right.
$$

where ${ }_{0}^{C} D_{\alpha}^{\psi}$ considered in this work is in the sense of Caputo fractional derivative with respect to a function $\psi$ which is more general than the classic Caputo fractional derivative. $A$ is the infinitesimal generator of a uniformly bounded $C_{0}$-semigroup $\{S(t)\}_{t \geq 0}$ on a Banach space $X, f:[0,+\infty) \times X \rightarrow X$ is a given function satisfying some assumptions.

Stochastic differential equations have a wide variety of applications in many fields such as economics, finance, engineering, and social sciences, thus they are viewed as better tools for describing the real-life phenomena than ordinary differential equations since noise or stochastic perturbation is unavoidable in nature as well as in man-made systems, see $[4,6,7,22,31,32]$. Recently, the stochastic differential equations driven by $\mathrm{fBm}$ have been investigated by many authors, see $[5,13,15,19,20,23,30,36]$ and the references therein.

However, it should be stressed that the existence uniqueness of mild solutions for $\psi$ Caputo fractional stochastic evolution equations is fairly scarce in contrast with the classical Caputo fractional stochastic evolution equations. This is greatly attributed to the relatively poor understanding of $\psi$-Caputo fractional derivative. In addition, many works focused on fractional stochastic evolution equations through various fixed point theorems when the corresponding semigroups are compact, which is convenient to obtain the corresponding compact resolvent operators. But for the case that the corresponding semigroups are noncompact, there are few results. Especially, there are no results considering the $\psi$-Caputo fractional stochastic differential equations driven by $\mathrm{fBm}$. Therefore, inspired by the above discussions, the scope of this work is to study the existence uniqueness of mild solutions for the following $\psi$-Caputo fractional stochastic evolution equations with varying-time delay driven by $\mathrm{fBm}$ with the corresponding semigroup be compact or not:

$$
\left\{\begin{array}{l}
{ }_{0}^{C} D_{\alpha}^{\psi} x(t)=A x(t)+f(t, x(t-r(t)))+\sigma(t) \frac{d B_{Q}^{H}(t)}{d t}, \quad t \in J=[0, b], \\
x(t)=\phi(t), \quad t \in[-\tau, 0]
\end{array}\right.
$$

where ${ }_{0}^{C} D_{\alpha}^{\psi}$ is $\psi$-Caputo fractional derivative of order $\frac{1}{2}<\alpha \leq 1 ; x(\cdot)$ takes values in a separable Hilbert space $X ; A: D(A) \subset X \rightarrow X$ is the infinitesimal generator of a $C_{0}$ semigroup $\{S(t)\}_{t \geq 0}$ on a real separable Hilbert space $X$. Let $Y$ be another separable Hilbert space. Let $\mathcal{L}(X, Y)$ denote the space of all bounded linear operators from $X$ to $Y$. For convenience, we use the notation $\|\cdot\|$ to denote the norms in $X, Y$ and $\mathcal{L}(X, Y)$ when no confusion possibly arises. $f: J \times X \rightarrow X$ is a function satisfying some specific assumptions given in $\left(\mathrm{H}_{2}\right) . B^{H}$ is a fractional Brownian motion with Hurst parameter $H \in\left(\frac{1}{2}, 1\right)$. The initial data $\phi \in C\left([-\tau, 0], \mathcal{L}_{2}(\Omega, X)\right)$, where $\mathcal{L}_{2}(\Omega, X)$ denote the collection of all stronglymeasurable, square-integrable, $X$-valued random variables. Obviously, $\mathcal{L}_{2}(\Omega, X)$ is a Banach space equipped with the norm $\|x(\cdot)\|_{\mathcal{L}_{2}(\Omega, X)}=(E|x(\cdot)|)^{\frac{1}{2}}$. Let $\mathcal{C}:=C\left([-r, b], \mathcal{L}_{2}(\Omega, X)\right)$ 
be the Banach space of all continuous functions $\xi$ from $[-\tau, b]$ into $\mathcal{L}_{2}(\Omega, X)$, equipped with the supremum norm $\|\xi\|_{\mathcal{C}}=\sup _{y \in[-\tau, b]}\left(E\|\xi(y)\|^{2}\right)^{\frac{1}{2}}$. In the sequel, $\mathcal{L}_{2}^{0}(\Omega, X)$ denotes the space of $\mathcal{F}_{0}$-measurable, $X$-valued, and square integrable stochastic process.

The paper is organized in the following way. In Sect. 2, we give some notations and useful concepts about fBm and fractional calculus. Section 3 aims to establish the existence of mild solutions for system (1.2) with Hurst parameter $H \in(1 / 2,1)$ by using the fixed point theorem and noncompact measure. In Sect. 4, we give an example to illustrate the application of the obtained abstract results. The conclusion is given in Sect. 5 .

\section{Preliminaries}

In this section, we introduce some notations, definitions, preliminary facts for further convenience.

Before going further, we begin by recalling some basic facts about $\mathrm{fBm}$ and Wiener integral with respect to $\mathrm{fBm}$.

Let $(\Omega, \mathcal{F}, P)$ be a complete probability space. Consider a time interval $[0, b]$ with arbitrary fixed horizon $b$, and let $\left\{B^{H}(t), t \in J\right\}$ be one-dimensional $\mathrm{fBm}$ with Hurst parameter $H \in(0,1)$. This means by definition that $B^{H}$ is a continuous centered Gaussian process with covariance function

$$
R_{H}(s, t)=\frac{1}{2}\left(t^{2 H}+s^{2 H}-|t-s|^{2 H}\right) .
$$

In the rest of the paper, we always assume $\frac{1}{2}<H<1$. Consider the square integrable kernel given by

$$
K_{H}(s, t)=c_{H} s^{\frac{1}{2}-H} \int_{s}^{t}(u-s) u^{H-\frac{1}{2}} d u,
$$

where $c_{H}=\left[\frac{H(2 H-1)}{\beta\left(2-2 H, H-\frac{1}{2}\right)}\right]^{\frac{1}{2}}, t>s, \beta(\cdot, \cdot)$ denotes the beta function. We take $K_{H}(s, t)=0$ for $t \leq s$, then it is easy to verify that

$$
\frac{\partial K_{H}}{\partial t}(t, s)=c_{H}\left(\frac{t}{s}\right)^{H-\frac{1}{2}}(t-s)^{H-\frac{3}{2}}
$$

We now consider an $\mathrm{fBM}\left\{B^{H}(t), t \in[0, b]\right\}$. We denote by $\xi$ the set of step functions on $[0, b]$. Let $\mathcal{H}$ be a Hilbert space defined as the closure of $\xi$ with respect to the scalar product

$$
\left\langle 1_{[0, t]}, 1_{[0, s]}\right\rangle_{H}=R_{H}(t, s) .
$$

The mapping $1_{[0, t]} \rightarrow\left\{B^{H}(t)\right\}$ can be extended to an isometry between $\mathcal{H}$ and the first Wiener chaos of the $\mathrm{fBm} \overline{\operatorname{span}}{ }^{L^{2}(\Omega)}\left\{B^{H}(t), t \in J\right\}$, and we will denote by $B^{H}(\varphi)$ the image of $\varphi$ under this isometry.

Let us define the linear operator $K_{H}^{*}$ from $\xi$ to $L^{2}([0, b])$ by

$$
\left(K_{H}^{*} \varphi\right)(s)=\int_{s}^{t} \varphi(t) \frac{\partial K_{H}}{\partial t}(t, s) d t
$$

Then $K_{H}^{*}$ is an isometry between $\mathcal{H}$ and $L^{2}([0, b])$. 
Consider the process $\omega=\omega(t), t \in[0, b]$ defined by

$$
\left.\omega(t)=B^{H}\left(\left(K_{H}^{*}\right)^{-1} 1_{[0, b]}\right)\right) .
$$

Then $\omega$ is a Wiener process, and $B^{H}$ has the integral representation

$$
B^{H}(t)=\int_{0}^{t} K_{H}(t, s) d \omega(s)
$$

Assume that there exists a complete orthogonal system $\left\{e_{n}\right\}_{n=1}^{\infty}$ in $Y$. Let $Q \in L(Y, Y)$ be an operator with finite trace $\operatorname{tr} Q=\sum_{n=1}^{\infty} \lambda_{n}<\infty\left(\lambda_{n} \geq 0\right)$ such that $Q e_{n}=\lambda_{n} e_{n}$. The infinite dimensional $\mathrm{fBM}$ on $Y$ can be defined by using covariance operator $Q$ as

$$
B^{H}(t)=B_{Q}^{H}(t)=\sum_{n=1}^{\infty} \sqrt{\lambda_{n}} \varphi e_{n} B_{n}^{H}(t)
$$

where $B_{n}^{H}(t)$ are one-dimensional standard fractional Brownian motions mutually independent on $(\Omega, \mathcal{F}, P)$. Consider the space $\mathcal{L}_{2}^{0}:=\mathcal{L}_{2}^{0}(Y, X)$ of all $Q$-Hilbert-Schmidt operators $\varphi: Y \rightarrow X$. We recall that $\varphi \in L(Y, X)$ is called a $Q$-Hilbert-Schmidt operator if

$$
\|\varphi\|_{\mathcal{L}_{2}^{0}}^{2}:=\sum_{n=1}^{\infty}\left\|\sqrt{\lambda_{n}} \varphi e_{n}\right\|^{2}<\infty
$$

and that the space $\mathcal{L}_{2}^{0}$ equipped with the inner product $\langle\varphi, \psi\rangle_{\mathcal{L}_{2}^{0}}=\sum_{n=1}^{\infty}\left\langle\varphi e_{n}, \psi e_{n}\right\rangle$ is a separable Hilbert space.

Let $(\phi(s))_{s \in[0, b]}$ be a deterministic function with values in $\mathcal{L}_{2}^{0}(Y, X)$. The stochastic integral of $\phi$ with respect to $B^{H}$ is defined by

$$
\int_{0}^{t} \phi(s) d B^{H}(s)=\sum_{n=1}^{\infty} \int_{0}^{t} \sqrt{\lambda_{n}}\left(K_{H}^{*}\left(\phi e_{n}\right)\right)(s) d B_{n}(s)
$$

Now we state some essential facts of fractional operators and Kuratowski's measure.

Definition 2.1 ([17] $\psi$-Riemann-Liouville fractional integral) Let $\alpha>0, f$ be an integrable function defined on $[a, b]$ and $\psi \in C^{1}([a, b])$ be an increasing function with $\psi^{\prime}(t) \neq 0$ for all $t \in[a, b]$. The $\psi$-Riemann-Liouville fractional integral operator of order $\alpha$ of a function $f$ is defined by

$$
\left({ }_{a} I_{\psi}^{\alpha} f\right)(t)=\frac{1}{\Gamma(\alpha)} \int_{a}^{t}(\psi(t)-\psi(s))^{\alpha-1} f(s) \psi^{\prime}(s) d s .
$$

It is obvious that when $\psi(t)=t,(2.2)$ is the classical Riemann-Liouville fractional operator. When $\psi(t)=\ln t,(2.2)$ is the Hadamard fractional operator.

Lemma 2.1 ([13]) If $\varphi:[0, b] \rightarrow \mathcal{L}_{2}^{0}(Y, X)$ satisfies $\int_{0}^{b}\|\varphi(s)\|_{\mathcal{L}_{2}^{0}}^{2}<\infty$, then the aforementioned sum in (2.1) is well defined as an $X$-valued random variable, and we have

$$
E\left\|\int_{0}^{t} \varphi(s) d B^{H}(s)\right\|^{2} \leq c_{0} H(2 H-1) t^{2 H-1} \int_{0}^{t}\|\varphi(s)\|_{\mathcal{L}_{2}^{0}}^{2} d s .
$$


Definition 2.2 ([17] ( $\psi$-Riemann-Liouville fractional derivative)) Let $n-1<\alpha<n, f$ be an integrable function defined on $[a, b]$ and $\psi \in C^{1}([a, b])$ be an increasing function with $\psi^{\prime}(t) \neq 0$ for all $t \in[a, b]$. The $\psi$-Riemann-Liouville fractional derivative of order $\alpha$ of a function $f$ is defined by

$$
\left({ }_{a} D_{\psi}^{\alpha} f\right)(t)=\left(\frac{1}{\psi^{\prime}(t)} \frac{d}{d t}\right)^{n}\left({ }_{a} I_{\psi}^{n-\alpha} f\right)(t)=\frac{\left(\frac{1}{\psi^{\prime}(t)} \frac{d}{d t}\right)^{n}}{\Gamma(n-\alpha)} \int_{a}^{t}(\psi(t)-\psi(s))^{n-\alpha-1} f(s) d s,
$$

where $n=\alpha+1$.

From the definition, when $\alpha=n \in \mathbb{N}$, we have $\left({ }_{a} D_{\psi}^{\alpha} f\right)(t)=\left(\frac{1}{\psi^{\prime}(t)} \frac{d}{d t}\right)^{n} f(t)$.

Lemma $2.2([2])$

(i) ${ }_{a} I_{\psi}^{\alpha}(\psi(x)-\psi(a))^{\beta-1}(t)=\frac{\Gamma(\beta)}{\Gamma(\beta+\alpha)}(\psi(t)-\psi(a))^{\beta+\alpha-1}$;

(ii) ${ }_{a} D_{\psi}^{\alpha}(\psi(x)-\psi(a))^{\beta-1}(t)=\frac{\Gamma(\beta)}{\Gamma(\beta-\alpha)}(\psi(t)-\psi(a))^{\beta+\alpha-1}$.

Definition $2.3\left(\left(\psi\right.\right.$-Caputo fractional derivative [17] )) Let $n-1<\alpha<n, f \in C^{n}([a, b])$ and $\psi \in C^{n}([a, b])$ be an increasing function with $\psi^{\prime}(t) \neq 0$ for all $t \in[a, b]$. The $\psi$-Caputo fractional derivative of order $\alpha$ of a function $f$ is defined by

$$
\left({ }_{a}^{C} D_{\psi}^{\alpha} f\right)(t)=\left({ }_{a} I_{\psi}^{n-\alpha} f^{[n]}\right)(t)=\frac{1}{\Gamma(n-\alpha)} \int_{a}^{t}(\psi(t)-\psi(s))^{n-\alpha-1} f^{[n]}(s) \psi^{\prime}(s) d s,
$$

where $n=[\alpha]+1$ and $f^{[n]}(t):=\left(\frac{1}{\psi^{\prime}(t)} \frac{d}{d t}\right)^{n} f(t)$ on $[a, b]$.

From the definition, it is clear that, when $\alpha=n \in \mathbb{N}$,

$$
{ }_{a}^{C} D_{\psi}^{\alpha} f(t)=f^{[n]}(t) .
$$

Theorem 2.1 ([2]) Let $f \in C^{n}([a, b])$ and $\alpha>0$. Then we have

$$
{ }_{a} I_{\psi}^{\alpha}\left({ }_{a}^{C} D_{\psi}^{\alpha} f(t)\right)=f(t)-\sum_{k=0}^{n-1} \frac{f^{[k]}\left(a^{+}\right)}{k !}(\psi(t)-\psi(a))^{k} .
$$

In particular, given $\alpha \in(0,1)$, we have ${ }_{a} I_{\psi}^{\alpha}\left({ }_{a}^{C} D_{\psi}^{\alpha} f(t)\right)=f(t)-f(a)$.

Definition 2.4 ([32]) Let $X$ be a Banach space and $\Omega_{x}$ be the bounded set of $X$. The Kuratowski measure of noncompactness is the map $\alpha: \Omega_{x} \rightarrow[0, \infty)$ defined by $\alpha(D)=\inf \{d>$ $0: D \subseteq \bigcup_{i=1}^{n} D_{i}$ and $\left.\operatorname{diam}\left(D_{i}\right) \leq d\right\}$, here $D \in \Omega_{x}$.

Lemma 2.3 ([33]) The noncompact measure $\alpha(\cdot)$ satisfies:

(i) for all bounded subsets $D_{1}, D_{2}$ of $X, D_{1} \subseteq D_{2}$ implies $\alpha\left(D_{1}\right) \leq \alpha\left(D_{2}\right)$;

(ii) $\alpha\{\{x\} \cup D\}=\alpha(D)$ for every $x \in X$ and every nonempty subset $D \in X$;

(iii) $\alpha\left(D_{1}\right)=0$ if and only if $D_{1}$ is relatively compact in $X$;

(iv) $\alpha\left(D_{1}+D_{2}\right) \leq \alpha\left(D_{1}\right)+\alpha\left(D_{2}\right)$, where $D_{1}+D_{2}=\left\{x+y: x \in D_{1}, y \in D_{2}\right\}$;

(v) $\alpha\left(D_{1} \cup D_{2}\right) \leq \max \left\{\alpha\left(D_{1}\right), \alpha\left(D_{2}\right)\right\}$;

(vi) $\alpha(\lambda D) \leq|\lambda| \alpha(D)$ for any $\lambda \in \mathbb{R}$;

(vii) $\alpha(U+x)=\alpha(U)$ for any $x \in X$;

(viii) If the map $Q: D(Q) \subset H \rightarrow X$ is Lipschitz continuous with constant $k$, then $\alpha(Q(S)) \leq k \alpha(S)$ for any bounded subset $S \subset D(Q)$, where $X$ is a Banach space. 
For any $W \in C(I, X)$, we define $\int_{0}^{t} W(s) d s=\left\{\int_{0}^{t} u(s) d s: u \in W\right\}$ for $t \in J$, where $W(s)=$ $\{u(s) \in X: u \in W\}$.

We use $\alpha(\cdot)$ and $\alpha_{C}(\cdot)$ to denote the Kuratowski measure of noncompactness on the bounded set of $X$ and $C([-\tau, b], X)$, respectively. For any $D \subset C([-\tau, b], X)$ and $t \in[0, b]$, set $D(t)=\{u(t) \mid u \in D\}$, then $D(t) \subset X$. If $D \subset C([-\tau, b], X)$ is bounded, then $D(t)$ is bounded in $X$ and $\alpha(D(t)) \leq \alpha_{C}(D)$.

Lemma 2.4 ([33]) Let $D \subset C([-\tau, b], X)$ be bounded and equicontinuous. Then $\alpha(D(t))$ is continuous on $[-\tau, b]$, and $\alpha_{C}(D)=\max _{t \in[-\tau, b]} \alpha(D(t))$.

Lemma 2.5 ([33]) Let $X$ be a Banach space, and let $D \subset X$ be bounded. Then there exists a countable set $D_{0} \subset D$ such that $\alpha(D) \leq 2 \alpha\left(D_{0}\right)$.

Lemma 2.6 ([14]) Let $X$ be a Banach space. If $D=\left\{u_{n}\right\}_{n=1}^{\infty} \subset C([-\tau, b], X)$ is a countable set and there exists a function $m \in L^{1}\left([-\tau, b], \mathbb{R}^{+}\right)$such that, for every $n \subset \mathbb{N}$,

$$
\left\|u_{n}(t)\right\| \leq m(t), \quad \text { a.e. } t \in[-\tau, b] .
$$

Then $\alpha(D(t))$ is Lebesgue integral on $[-\tau, b]$, and

$$
\alpha\left(\left\{\int_{0}^{b} u_{n}(t) d t \mid n \in \mathbb{N}\right\}\right) \leq 2 \int_{0}^{b} \alpha(D(t)) d t
$$

Lemma 2.7 ((Sadovskii fixed point theorem [18])) Let $X$ be a Banach space. Assume that $D \subset X$ is a bounded closed and convex set on $X$ and $Q: D \rightarrow D$ is a condensing operator. Then $Q$ has at least one fixed point in D.

For $C_{0}$ semigroup $\{S(t)\}_{t \geq 0}$, the following property will be used:

There is $M \geq 1$ such that

$$
M:=\sup _{t \in[0,+\infty)} S(t)<\infty
$$

Lemma 2.8 The system

$$
\left\{\begin{array}{l}
{ }_{0}^{C} D_{\alpha}^{\psi} x(t)=A x(t)+f(t, x(t-r(t)))+\sigma(t) \frac{d B_{Q}^{H}(t)}{d t}, \quad t \in J, \\
x(t)=\phi(t), \quad t \in[-\tau, 0],
\end{array}\right.
$$

is equivalent to the integral equation

$$
\begin{aligned}
x(t)= & \phi(0)+\frac{1}{\Gamma(\alpha)} \int_{0}^{t}(\psi(t)-\psi(s))^{\alpha-1}[A x(s)+f(s, x(s-r(s)))] \psi^{\prime}(s) d s \\
& +\frac{1}{\Gamma(\alpha)} \int_{0}^{t}(\psi(t)-\psi(s))^{\alpha-1} \psi^{\prime}(s) \sigma(s) d B_{Q}^{H}(s), \quad t \in J .
\end{aligned}
$$

Proof We can readily obtain the result from Definition 2.3 and Theorem 2.1. Here we omit it. 


\section{Lemma 2.9 If}

$$
\begin{aligned}
x(t)= & \phi(0)+\frac{1}{\Gamma(\alpha)} \int_{0}^{t}(\psi(t)-\psi(s))^{\alpha-1}[A x(s)+f(s, x(s-r(s)))] \psi^{\prime}(s) d s \\
& +\frac{1}{\Gamma(\alpha)} \int_{0}^{t}(\psi(t)-\psi(s))^{\alpha-1} \psi^{\prime}(s) \sigma(s) d B_{Q}^{H}(s),
\end{aligned}
$$

then we have

$$
\begin{aligned}
x(t)= & S_{\alpha}^{\psi}(t, 0) \phi(0)+\int_{0}^{t}(\psi(t)-\psi(s))^{\alpha-1} T_{\alpha}^{\psi}(t, s) f\left(s, x(s-r(s)) \psi^{\prime}(s) d s\right. \\
& +\int_{0}^{t}(\psi(t)-\psi(s))^{\alpha-1} T_{\alpha}^{\psi}(t, s) \sigma(s) \psi^{\prime}(s) d B_{Q}^{H}(s),
\end{aligned}
$$

where

$$
\begin{aligned}
& S_{\alpha}^{\psi}(t, s) x=\int_{0}^{\infty} \phi_{\alpha}(\theta) S\left((\psi(t)-\psi(s))^{\alpha} \theta\right) x d \theta, \\
& T_{\alpha}^{\psi}(t, s) x=\alpha \int_{0}^{\infty} \theta \phi_{\alpha}(\theta) T\left((\psi(t)-\psi(s))^{\alpha} \theta\right) x d \theta,
\end{aligned}
$$

$\phi_{\alpha}$ is the probability density function defined on $(0, \infty)$, that is, $\phi_{\alpha}(\theta) \geq 0, \theta \in(0, \infty)$ and $\int_{0}^{\infty} \phi_{\alpha}(\theta) d \theta=1$.

Proof The proof is similar to the proof of Lemma 3.1 in [2], we can obtain the result by doing the necessary adjustments. We omit it here.

Lemma 2.10 ([2]) The operators $S_{\alpha}^{\psi}$ and $T_{\alpha}^{\psi}$ have the following properties:

(i) For any fixed $t \geq s \geq 0, S_{\alpha}^{\psi}(t, s)$ and $T_{\alpha}^{\psi}(t, s)$ are bounded linear operators with $\left\|S_{\alpha}^{\psi}(t, s)(x)\right\| \leq M\|x\|$ and $\left\|T_{\alpha}^{\psi}(t, s)(x)\right\| \leq \frac{M}{\Gamma(\alpha)}\|x\|$ for all $x \in X$.

(ii) The operators $S_{\alpha}^{\psi}$ and $T_{\alpha}^{\psi}$ are strongly continuous for all $t \geq s \geq 0$, that is, for every $x \in X$ and $0 \leq s \leq t_{1}<t_{2} \leq b$, we have

$$
\left\|S_{\alpha}^{\psi}\left(t_{2}, s\right) x-S_{\alpha}^{\psi}\left(t_{1}, s\right) x\right\| \rightarrow 0
$$

and

$$
\left\|T_{\alpha}^{\psi}\left(t_{2}, s\right) x-T_{\alpha}^{\psi}\left(t_{1}, s\right) x\right\| \rightarrow 0
$$

as $t_{1}-t_{2} \rightarrow 0$.

\section{Main results}

In this section, we present and prove the existence of mild solutions for system (1.2). To develop our results, we first give the concept of mild solution for system (1.2).

Definition 3.1 An $\mathcal{F}_{t}$-adapted and measurable stochastic process $x \in \mathcal{L}_{2}^{0}(\Omega, X)$ is said to be a mild solution of system (1.2) if

(1) $x(t)$ is measurable, $\mathcal{F}_{t}$-adapted, and has càdlàg path on $0 \leq t \leq b$ almost everywhere; 
(2) for $t \in[-\tau, 0], x(t)=\phi(t)$;

(3) for each $0 \leq t \leq b, x(t)$ satisfies the following integral equation:

$$
\begin{aligned}
x(t)= & S_{\alpha}^{\psi}(t, 0) \phi(0)+\int_{0}^{t}(\psi(t)-\psi(s))^{\alpha-1} T_{\alpha}^{\psi}(t, s) f\left(s, x(s-r(s)) \psi^{\prime}(s) d s\right. \\
& +\int_{0}^{t}(\psi(t)-\psi(s))^{\alpha-1} T_{\alpha}^{\psi}(t, s) \sigma(s) \psi^{\prime}(s) d B_{Q}^{H}(s) .
\end{aligned}
$$

For further convenience, set $\frac{M^{2}}{\Gamma^{2}(\alpha)} \psi^{\prime}(b) \frac{(\psi(b)-\psi(0))^{2 \alpha-1}}{2 \alpha-1}=M_{1}$.

Before stating and proving the main results, we introduce the following hypotheses:

$\left(\mathrm{H}_{0}\right)$ Semigroup $S(t)$ is compact for each $t>0$;

$\left(\mathrm{H}_{1}\right)$ Function $\psi(t) \in C^{2}(J, \mathbb{R})$ and $\psi^{\prime \prime}(t)>0, \psi^{\prime}(t)>0$ for $\forall t \in J$;

$\left(\mathrm{H}_{2}\right)$ (2a) For each $x \in X$, the function $f(\cdot, x): J \rightarrow X$ is strongly measurable with respect to $t$, and for each $t \in J$, the function $f(t, \cdot): X \rightarrow X$ is continuous with respect to $x$;

(2b) There exist a continuous nondecreasing function $\mu:[0, \infty) \rightarrow(0, \infty)$ and constant $L$ such that, for any $(t, x) \in J \times X$, we have

$$
E\|f(t, x(t-r(t)))\|^{2} \leq L\left(1+\mu\left(\|x\|_{\mathcal{C}}^{2}\right)\right), \quad \lim _{r \rightarrow \infty} \inf \frac{\mu(r)}{r}=\Lambda<\infty ;
$$

$\left(\mathrm{H}_{3}\right)$ The function $\sigma: J \rightarrow \mathcal{L}_{2}^{0}(X, Y)$ satisfies

$$
\sup _{t \in J}\|\sigma(s)\|_{\mathcal{L}_{2}^{0}}^{2}<\infty, \quad \forall t \in J
$$

We define the operator $\Phi: \mathcal{C} \rightarrow \mathcal{C}$ as follows:

$$
\begin{aligned}
(\Phi x)(t)= & S_{\alpha}^{\psi}(t, 0) \phi(0)+\int_{0}^{t}(\psi(t)-\psi(s))^{\alpha-1} T_{\alpha}^{\psi}(t, s) f\left(s, x(s-r(s)) \psi^{\prime}(s) d s\right. \\
& +\int_{0}^{t}(\psi(t)-\psi(s))^{\alpha-1} T_{\alpha}^{\psi}(t, s) \sigma(s) \psi^{\prime}(s) d B_{Q}^{H}(s) .
\end{aligned}
$$

According to assumptions $\left(\mathrm{H}_{1}\right)$ and $\left(\mathrm{H}_{2}\right)$ and Lemma 2.10, we can obtain

$$
\begin{aligned}
& E\left\|\int_{0}^{t}(\psi(t)-\psi(s))^{\alpha-1} T_{\alpha}^{\psi}(t, s) f(s, x(s-r(s))) \psi^{\prime}(s) d s\right\|^{2} \\
& \quad \leq \frac{M^{2}}{\Gamma^{2}(\alpha)} E\left\|\int_{0}^{t}(\psi(t)-\psi(s))^{\alpha-1} f(s, x(s-r(s))) \psi^{\prime}(s) d s\right\|^{2} \\
& \quad \leq \frac{M^{2}}{\Gamma^{2}(\alpha)} \int_{0}^{t} E\left\|(\psi(t)-\psi(s))^{\alpha-1} f(s, x(s-r(s))) \psi^{\prime}(s)\right\|^{2} d s \\
& \quad \leq \frac{M^{2}}{\Gamma^{2}(\alpha)} \int_{0}^{t}(\psi(t)-\psi(s))^{2 \alpha-2} E\|f(s, x(s-r(s)))\|^{2}\left(\psi^{\prime}(s)\right)^{2} d s \\
& \quad \leq \frac{M^{2}}{\Gamma^{2}(\alpha)} \psi^{\prime}(b) \frac{(\psi(t)-\psi(s))^{2 \alpha-1}}{2 \alpha-1} L\left(1+\mu\left(\|x\|_{\mathcal{C}}^{2}\right)\right. \\
& \quad \leq \frac{M^{2}}{\Gamma^{2}(\alpha)} \psi^{\prime}(b) \frac{(\psi(b)-\psi(0))^{2 \alpha-1}}{2 \alpha-1} L\left(1+\mu\left(\|x\|_{\mathcal{C}}^{2}\right) .\right.
\end{aligned}
$$


Now, from assumptions $\left(\mathrm{H}_{1}\right)$ and $\left(\mathrm{H}_{3}\right)$, we can get

$$
\begin{aligned}
& E\left\|\int_{0}^{t}(\psi(t)-\psi(s))^{\alpha-1} T_{\alpha}^{\psi}(t, s) \sigma(s) \psi^{\prime}(s) d B_{Q}^{H}(s)\right\|^{2} \\
& \quad \leq \frac{M^{2}}{\Gamma^{2}(\alpha)} E\left\|\int_{0}^{t}(\psi(t)-\psi(s))^{\alpha-1} \sigma(s) \psi^{\prime}(s) d B_{Q}^{H}(s)\right\|^{2} \\
& \quad \leq c_{0} H(2 H-1) t^{2 H-1} \frac{M^{2}}{\Gamma^{2}(\alpha)} \int_{0}^{t} E\left\|(\psi(t)-\psi(s))^{\alpha-1} \sigma(s) \psi^{\prime}(s)\right\|^{2} d s \\
& \quad \leq c_{0} H(2 H-1) t^{2 H-1} \frac{M^{2}}{\Gamma^{2}(\alpha)} \int_{0}^{t}(\psi(t)-\psi(s))^{2 \alpha-2} E\|\sigma(s)\|^{2}\left(\psi^{\prime}(s)\right)^{2} d s \\
& \quad \leq c_{0} H(2 H-1) b^{2 H-1} \frac{M^{2}}{\Gamma^{2}(\alpha)} \psi^{\prime}(b) \frac{(\psi(t)-\psi(s))^{2 \alpha-1}}{2 \alpha-1} \sup _{t \in J}\|\sigma(s)\|_{\mathcal{L}_{2}^{0}}^{2} \\
& \quad \leq c_{0} H(2 H-1) b^{2 H-1} \frac{M^{2}}{\Gamma^{2}(\alpha)} \psi^{\prime}(b) \frac{(\psi(b)-\psi(0))^{2 \alpha-1}}{2 \alpha-1} \sup _{t \in J}\|\sigma(s)\|_{\mathcal{L}_{2}^{0}}^{2} .
\end{aligned}
$$

In the following, we give the first existence result for system (1.2) with the corresponding semigroup be compact.

Theorem 3.1 Suppose that hypotheses $\left(H_{0}\right)-\left(H_{3}\right)$ hold, then system (1.2) has at least one mild solution defined on $J$ provided that $3 M_{1} L \Lambda<1$.

Proof Denote $B_{q}=\left\{x \in \mathcal{C},\|x\|_{\mathcal{C}}^{2} \leq q\right\}$, obviously, $B_{q}$ is a bounded, closed, convex set in $\mathcal{C}$. We divide the proof into three steps.

Step 1. We shall show that there exists a constant $r=r(a)$ such that $\Phi\left(B_{r}\right) \subset B_{r}$.

In fact, if it is not true, then for each positive constant $r$ there exists some $\hat{x} \in B_{r}$ such that $\Phi(\hat{x}) \notin B_{r}$, i.e.,

$$
\begin{aligned}
r< & E\|\Psi(\hat{x})\|^{2} \\
\leq & 3 E\left\|S_{\alpha}^{\psi}(t, 0) \phi(0)\right\|^{2} \\
& +3 E\left\|\int_{0}^{t}(\psi(t)-\psi(s))^{\alpha-1} T_{\alpha}^{\psi}(t, s) \psi^{\prime}(s) f(s, \hat{x}(s-r(s))) d s\right\|^{2} \\
& +3 E\left\|\int_{0}^{t}(\psi(t)-\psi(s))^{\alpha-1} T_{\alpha}^{\psi}(t, s) \psi^{\prime}(s) \sigma(s) d B_{Q}^{H}(s)\right\|^{2} \\
\leq & 3 M^{2} \phi^{2}(0)+3 \frac{M^{2}}{\Gamma^{2}(\alpha)} \psi^{\prime}(b) \frac{(\psi(b)-\psi(0))^{2 \alpha-1}}{2 \alpha-1} L\left(1+\mu\left(\|\hat{x}\|_{\mathcal{C}}^{2}\right)\right. \\
& +3 c_{0} H(2 H-1) b^{2 H-1} \frac{M^{2}}{\Gamma^{2}(\alpha)} \psi^{\prime}(b) \frac{(\psi(b)-\psi(0))^{2 \alpha-1}}{2 \alpha-1} \sup _{t \in J}\|\sigma(s)\|_{\mathcal{L}_{2}^{0}}^{2}
\end{aligned}
$$

Dividing both sides by $r$ and taking $r \rightarrow \infty$, we get

$$
3 \frac{M^{2}}{\Gamma^{2}(\alpha)} \psi^{\prime}(b) \frac{(\psi(b)-\psi(0))^{2 \alpha-1}}{2 \alpha-1} L \Lambda=3 M_{1} L \Lambda>1
$$

which is a contradiction to the hypotheses of Theorem 3.1. Thus, there exists $r$ such that $\Phi$ maps $B_{r}$ into itself. 
Step 2. $\Phi$ is continuous on $B_{r}$.

For any $x_{n}, x \in B_{r}, n=1,2, \ldots$, with $\lim _{n \rightarrow \infty}\left\|x_{n}-x\right\|_{\mathcal{C}}^{2}=0$, we get $\lim _{n \rightarrow \infty} x_{n}(t)=x(t)$ for $t \in J$. Thus, by assumption $\left(\mathrm{H}_{2}\right)$, we can easily get $\Phi$ is continuous on $B_{r}$.

Step 3. $\Phi$ is a completely continuous operator.

We subdivide Step 3 into three claims.

Claim 1. $\Phi$ maps bounded sets into uniformly bounded sets in $\mathcal{C}$.

Actually, we only need to show that there exists a positive constant $\Delta$ such that, for each $x \in B_{r}$, one has $\|\Phi x\|_{\mathcal{C}} \leq \Delta$. As a matter of fact, for each $t \in J$, Step 1 enables us to obtain this assertion.

Claim 2. $\Phi\left(B_{r}\right)$ is equicontinuous on $B_{r}$.

For $\forall x \in B_{r}$, let $0=t_{1}<t_{2} \leq b$. Taking $\left(\mathrm{H}_{2}\right),\left(\mathrm{H}_{3}\right)$ and the strong continuity of $\left\{S_{\alpha}^{\psi}(t)\right\}_{t \geq 0}$ into account, we get as $t_{2} \rightarrow 0$,

$$
\begin{aligned}
& E\left\|(\Phi x)\left(t_{2}\right)-(\Phi x)(0)\right\|^{2} \\
& \leq 3 E\left\|S_{\psi}^{\alpha}\left(t_{2}, 0\right)-S_{\psi}^{\alpha}(0,0)\right\|^{2} \\
&+3 E\left\|\int_{0}^{t_{2}}\left(\psi\left(t_{2}\right)-\psi(s)\right)^{\alpha-1} T_{\alpha}^{\psi}\left(t_{2}-s\right) f(s, x(s-r(s))) \psi^{\prime}(s) d s\right\|^{2} \\
&+3 E\left\|\int_{0}^{t_{2}}\left(\psi\left(t_{2}\right)-\psi(s)\right)^{\alpha-1} T_{\alpha}^{\psi}\left(t_{2}-s\right) \sigma(s) \psi^{\prime}(s) d B_{Q}^{H}(s)\right\|^{2} \\
& \leq 3 E\left\|S_{\psi}^{\alpha}\left(t_{2}, 0\right)-S_{\psi}^{\alpha}(0,0)\right\|^{2} \\
&+3 \frac{M^{2}}{\Gamma^{2}(\alpha)} \frac{\left(\psi\left(t_{2}\right)-\psi(0)\right)^{2 \alpha-1}}{2 \alpha-1} \psi^{\prime}(b) L\left(1+\mu\left(\|x\|_{\mathcal{C}}^{2}\right)\right. \\
&+3 \frac{M^{2}}{\Gamma^{2}(\alpha)} \frac{\left(\psi\left(t_{2}\right)-\psi(0)\right)^{2 \alpha-1}}{2 \alpha-1} \psi^{\prime}(b)\|\sigma(s)\|_{\mathcal{L}_{2}^{0}}^{2} \rightarrow 0 .
\end{aligned}
$$

For $0<t_{1}<t_{2} \leq b$, from the strong continuity of $\left\{T_{\alpha}^{\psi}(t)\right\}_{t \geq 0}$, there exist arbitrarily small constants $\delta, \tau>0$ such that as long as $\left|t_{2}-t_{1}\right|<\delta$, we have $\left\|T_{\alpha}^{\psi}\left(t_{1}\right)-T_{\alpha}^{\psi}\left(t_{2}\right)\right\|<\tau$. Then, for $\forall x \in B_{r}$, we can obtain

$$
\begin{aligned}
& E\left\|(\Phi x)\left(t_{2}\right)-(\Phi x)\left(t_{1}\right)\right\|^{2} \\
& \leq 7 E\left\|S_{\psi}^{\alpha}\left(t_{2}, 0\right)-S_{\psi}^{\alpha}\left(t_{1}, 0\right)\right\|^{2} \\
& \quad+7 E \| \int_{0}^{t_{1}}\left[\left(\psi\left(t_{2}\right)-\psi(s)\right)^{\alpha-1}-\left(\psi\left(t_{1}\right)-\psi(s)\right)^{\alpha-1}\right] T_{\alpha}^{\psi}\left(t_{2}-s\right) \\
& \quad \times f(s, x(s-r(s))) \psi^{\prime}(s) d s \|^{2} \\
& \quad+7 E \| \int_{0}^{t_{1}}\left[\left(\psi\left(t_{2}\right)-\psi(s)\right)^{\alpha-1}-\left(\psi\left(t_{1}\right)-\psi(s)\right)^{\alpha-1}\right] T_{\alpha}^{\psi}\left(t_{2}-s\right) \\
& \quad \times \sigma(s) \psi^{\prime}(s) d B_{Q}^{H}(s) \|^{2} \\
& \quad+7 E \| \int_{0}^{t_{1}}\left(\psi\left(t_{1}\right)-\psi(s)\right)^{\alpha-1}\left[T_{\alpha}^{\psi}\left(t_{2}-s\right)-T_{\alpha}^{\psi}\left(t_{1}-s\right)\right] \\
& \quad \times f(s, x(s-r(s))) \psi^{\prime}(s) d s \|^{2}
\end{aligned}
$$




$$
\begin{aligned}
& +7 E\left\|\int_{0}^{t_{1}}\left(\psi\left(t_{1}\right)-\psi(s)\right)^{\alpha-1}\left[T_{\alpha}^{\psi}\left(t_{2}-s\right)-T_{\alpha}^{\psi}\left(t_{1}-s\right)\right] \sigma(s) \psi^{\prime}(s) d B_{Q}^{H}(s)\right\|^{2} \\
& +7 E\left\|\int_{t_{1}}^{t_{2}}\left(\psi\left(t_{2}\right)-\psi(s)\right)^{\alpha-1} T_{\alpha}^{\psi}\left(t_{2}-s\right) f(s, x(s-r(s))) \psi^{\prime}(s) d s\right\|^{2} \\
& +7 E\left\|\int_{t_{1}}^{t_{2}}\left(\psi\left(t_{2}\right)-\psi(s)\right)^{\alpha-1} T_{\alpha}^{\psi}\left(t_{2}-s\right) \sigma(s) \psi^{\prime}(s) d B_{Q}^{H}(s)\right\|^{2} \\
& \leq 7 E\left\|S_{\psi}^{\alpha}\left(t_{2}, 0\right)-S_{\psi}^{\alpha}\left(t_{1}, 0\right)\right\|^{2} \\
& +7 \int_{0}^{t_{1}}\left\|\left(\psi\left(t_{2}\right)-\psi(s)\right)^{\alpha-1}-\left(\psi\left(t_{1}\right)-\psi(s)\right)^{\alpha-1}\right\|^{2}\left\|T_{\alpha}^{\psi}\left(t_{2}-s\right)\right\|^{2} \\
& \times E\|f(s, x(s-r(s)))\|^{2}\left(\psi^{\prime}(s)\right)^{2} d s \\
& +7 \int_{0}^{t_{1}}\left\|\left(\psi\left(t_{2}\right)-\psi(s)\right)^{\alpha-1}-\left(\psi\left(t_{1}\right)-\psi(s)\right)^{\alpha-1}\right\|^{2}\left\|T_{\alpha}^{\psi}\left(t_{2}-s\right)\right\|^{2} \\
& \times\|\sigma(s)\|^{2}\left(\psi^{\prime}(s)\right)^{2} d B_{Q}^{H}(s) \\
& +7\left\|T_{\alpha}^{\psi}\left(t_{2}-s\right)-T_{\alpha}^{\psi}\left(t_{1}-s\right)\right\|^{2} \psi^{\prime}(b) \frac{(\psi(b))^{2 \alpha-1}}{2 \alpha-1} L\left(1+\mu\left(\|x\|_{\mathcal{C}}^{2}\right)\right) \\
& +7\left\|T_{\alpha}^{\psi}\left(t_{2}-s\right)-T_{\alpha}^{\psi}\left(t_{1}-s\right)\right\|^{2} \psi^{\prime}(b) \frac{(\psi(b))^{2 \alpha-1}}{2 \alpha-1} \sup _{t \in J}\|\sigma(s)\|_{\mathcal{L}_{2}^{0}}^{2} \\
& +7 \frac{M^{2}}{\Gamma^{2}(\alpha)} \int_{t_{1}}^{t_{2}}\left(t_{2}-s\right)^{2(\alpha-1)} E\|f(s, x(s-r(s)))\|^{2}\left(\psi^{\prime}(s)\right)^{2} d s \\
& +7 \frac{M^{2}}{\Gamma^{2}(\alpha)} \int_{t_{1}}^{t_{2}}\left(t_{2}-s\right)^{2(\alpha-1)}\|\sigma(s)\|_{\mathcal{L}_{2}^{0}}^{2}\left(\psi^{\prime}(s)\right)^{2} d B_{Q}^{H}(s):=7 \sum_{i=1}^{7} I_{i},
\end{aligned}
$$

where

$$
\begin{aligned}
I_{1}= & E\left\|S_{\psi}^{\alpha}\left(t_{2}, 0\right)-S_{\psi}^{\alpha}\left(t_{1}, 0\right)\right\|^{2}, \\
I_{2}= & \int_{0}^{t_{1}}\left\|\left(\psi\left(t_{2}\right)-\psi(s)\right)^{\alpha-1}-\left(\psi\left(t_{1}\right)-\psi(s)\right)^{\alpha-1}\right\|^{2}\left\|T_{\alpha}^{\psi}\left(t_{2}-s\right)\right\|^{2} \\
& \times E\|f(s, x(s-r(s)))\|^{2}\left(\psi^{\prime}(s)\right)^{2} d s, \\
I_{3}= & \int_{0}^{t_{1}}\left\|\left(\psi\left(t_{2}\right)-\psi(s)\right)^{\alpha-1}-\left(\psi\left(t_{1}\right)-\psi(s)\right)^{\alpha-1}\right\|^{2}\left\|T_{\alpha}^{\psi}\left(t_{2}-s\right)\right\|^{2} \\
& \times\|\sigma(s)\|^{2}\left(\psi^{\prime}(s)\right)^{2} d B_{Q}^{H}(s), \\
I_{4}= & \left\|T_{\alpha}^{\psi}\left(t_{2}-s\right)-T_{\alpha}^{\psi}\left(t_{1}-s\right)\right\|^{2} \psi^{\prime}(b) \frac{(\psi(b))^{2 \alpha-1}}{2 \alpha-1} L\left(1+\mu\left(\|x\|_{\mathcal{C}}^{2}\right)\right), \\
I_{5}= & \left\|T_{\alpha}^{\psi}\left(t_{2}-s\right)-T_{\alpha}^{\psi}\left(t_{1}-s\right)\right\|^{2} \psi^{\prime}(b) \frac{(\psi(b))^{2 \alpha-1}}{2 \alpha-1} \sup _{t \in J}\|\sigma(s)\|_{\mathcal{L}_{2}^{0},}^{2} \\
I_{6}= & \frac{M^{2}}{\Gamma^{2}(\alpha)} \int_{t_{1}}^{t_{2}}\left(\psi\left(t_{2}\right)-\psi(s)\right)^{2(\alpha-1)} E\|f(s, x(s-r(s)))\|^{2}\left(\psi^{\prime}(s)\right)^{2} d s, \\
I_{7}= & \frac{M^{2}}{\Gamma^{2}(\alpha)} \int_{t_{1}}^{t_{2}}\left(\psi\left(t_{2}\right)-\psi(s)\right)^{2(\alpha-1)}\|\sigma(s)\|_{\mathcal{L}_{2}^{0}}^{2}\left(\psi^{\prime}(s)\right)^{2} d B_{Q}^{H}(s) .
\end{aligned}
$$

We next verify if each term tends to 0 as $t_{2}-t_{1} \rightarrow 0$. 
For $I_{1}$, from the strong continuity of $\left\{S_{\alpha}^{\psi}(t)\right\}_{t \geq 0}$, we can draw the conclusion.

For $I_{2}$, letting $t_{2}-t_{1} \rightarrow 0$ leads to

$$
\begin{aligned}
I_{2} \leq & \int_{0}^{t_{1}}\left[\left(\psi\left(t_{2}\right)-\psi(s)\right)^{\alpha-1}-\left(\psi\left(t_{1}\right)-\psi(s)\right)^{\alpha-1}\right] \psi^{\prime}(s) d s \\
& \times \int_{0}^{t_{1}}\left[\left(\psi\left(t_{2}\right)-\psi(s)\right)^{\alpha-1}-\left(\psi\left(t_{1}\right)-\psi(s)\right)^{\alpha-1}\right]\left\|T_{\alpha}^{\psi}\left(t_{2}-s\right)\right\|^{2} \\
& \times E\|f(s, x(s-r(s)))\|^{2} \psi^{\prime}(s) d s \\
\leq & \frac{M^{2}}{\Gamma^{2}(\alpha)}\left(\frac{\left(\psi\left(t_{2}\right)-\psi\left(t_{1}\right)\right)^{\alpha}-\left(\psi\left(t_{2}\right)\right)^{\alpha}+\left(\psi\left(t_{1}\right)\right)^{\alpha}}{\alpha}\right)^{2} L\left(1+\mu\left(\|x\|_{\mathcal{C}}^{2}\right) \rightarrow 0 .\right.
\end{aligned}
$$

Similarly, we can get $I_{3}$ tends to zero as $t_{2}-t_{1} \rightarrow 0$.

The strong continuity of $\left\{T_{\alpha}^{\psi}(t)\right\}_{t \geq 0}$ leads to $\left\|T_{\alpha}^{\psi}\left(t_{2}-s\right)-T_{\alpha}^{\psi}\left(t_{1}-s\right)\right\|^{2} \rightarrow 0$ as $t_{2}-t_{1} \rightarrow 0$, thus $I_{4}, I_{5}$ tend to 0 as $t_{2}-t_{1} \rightarrow 0$.

In addition, we can derive that, as $t_{2}-t_{1} \rightarrow 0$,

$$
\begin{aligned}
I_{6} & \leq \frac{M^{2}}{\Gamma^{2}(\alpha)} \int_{t_{1}}^{t_{2}}\left(\psi\left(t_{2}\right)-\psi(s)\right)^{2(\alpha-1)} E\|f(s, x(s-v(s)))\|^{2}\left(\psi^{\prime}(s)\right)^{2} d s \\
& \leq \frac{M^{2}}{\Gamma^{2}(\alpha)} \psi^{\prime}(b) \frac{\left(\psi\left(t_{2}\right)-\psi\left(t_{1}\right)\right)^{2 \alpha-1}}{2 \alpha-1} L\left(1+\mu\left(\|x\|_{\mathcal{C}}^{2}\right) \rightarrow 0 .\right.
\end{aligned}
$$

Similarly, we can obtain $I_{7} \rightarrow 0$ as $t_{2}-t_{1} \rightarrow 0$.

Therefore, we derive that $\Phi$ is equicontinuous on $B_{r}$.

Claim 3. $V(t)=\left\{(\Phi x)(t), x \in B_{r}\right\}$ is relatively compact in $X$.

Let $0<t \leq b$ be fixed, for $\forall \lambda \in(0, t)$ and $\forall \delta>0, x \in B_{r}$, define an operator

$$
\begin{aligned}
\left(\Phi^{\lambda, \delta} x\right)(t) & \\
= & \int_{0}^{\infty} \phi_{\alpha}(\theta) S\left((\psi(t)-\psi(s))^{\alpha} \theta\right) \phi(0) d \theta \\
& +\alpha \int_{0}^{t-\lambda} \int_{\delta}^{\infty} \theta(\psi(t)-\psi(s))^{\alpha-1} \phi_{\alpha}(\theta) S\left((\psi(t)-\psi(s))^{\alpha} \theta\right) \\
& \times f(s, x(s-r(s))) \psi^{\prime}(s) d \theta d s \\
& +\alpha \int_{0}^{t-\lambda} \int_{\delta}^{\infty} \theta(\psi(t)-\psi(s))^{\alpha-1} \phi_{\alpha}(\theta) S\left((\psi(t)-\psi(s))^{\alpha} \theta\right) \\
& \times \sigma(s) \psi^{\prime}(s) d \theta d B_{Q}^{H}(s) \\
= & \int_{0}^{\infty} \phi_{\alpha}(\theta) S\left((\psi(t)-\psi(s))^{\alpha} \theta\right) \phi(0) d \theta \\
& +\alpha S\left(\lambda^{\alpha} \theta\right) \int_{0}^{t-\lambda} \int_{\delta}^{\infty} \theta(\psi(t)-\psi(s))^{\alpha-1} \phi_{\alpha}(\theta) S\left((\psi(t)-\psi(s))^{\alpha} \theta-\lambda^{\alpha} \theta\right) \\
& \times f(s, x(s-r(s))) \psi^{\prime}(s) d \theta d s \\
& +\alpha S\left(\lambda^{\alpha} \theta\right) \int_{0}^{t-\lambda} \int_{\delta}^{\infty} \theta(\psi(t)-\psi(s))^{\alpha-1} \phi_{\alpha}(\theta) S\left((\psi(t)-\psi(s))^{\alpha} \theta-\lambda^{\alpha} \theta\right) \\
& \times \sigma(s) \psi^{\prime}(s) d \theta d B_{Q}^{H}(s) .
\end{aligned}
$$


From the compactness of $S\left(\lambda^{\alpha} \delta\right), \lambda^{\alpha} \delta>0$, we obtain that, for $\forall \lambda \in(0, t)$ and $\forall \delta>0$, the set $V^{\varepsilon, \delta}(t)=\left\{\left(\Phi^{\lambda, \delta} x\right)(t), x \in B_{r}\right\}$ is relatively compact in $X$.

Moreover, for each $x \in B_{r}$, from $\left(\mathrm{H}_{1}\right)-\left(\mathrm{H}_{3}\right)$, we have

$$
\begin{aligned}
& E\left\|(\Phi x)(t)-\left(\Phi^{\lambda, \delta} x\right)(t)\right\|^{2} \\
& =E \| \int_{0}^{t} \int_{0}^{\delta} \alpha \theta(\psi(t)-\psi(s))^{\alpha-1} \phi_{\alpha}(\theta) S\left((\psi(t)-\psi(s))^{\alpha} \theta\right) \\
& \times f(s, x(s-r(s))) \psi^{\prime}(s) d s \\
& +\int_{0}^{t} \int_{\delta}^{\infty} \alpha \theta(\psi(t)-\psi(s))^{\alpha-1} \phi_{\alpha}(\theta) S\left((\psi(t)-\psi(s))^{\alpha} \theta\right) f(s, x(s-r(s))) \psi^{\prime}(s) d s \\
& +\int_{0}^{t} \int_{0}^{\delta} \alpha \theta(\psi(t)-\psi(s))^{\alpha-1} \phi_{\alpha}(\theta) S\left((\psi(t)-\psi(s))^{\alpha} \theta\right) \sigma(s) \psi^{\prime}(s) d B_{Q}^{H}(s) \\
& +\int_{0}^{t} \int_{\delta}^{\infty} \alpha \theta(\psi(t)-\psi(s))^{\alpha-1} \phi_{\alpha}(\theta) S\left((\psi(t)-\psi(s))^{\alpha} \theta\right) \sigma(s) \psi^{\prime}(s) d B_{Q}^{H}(s) \\
& -\int_{0}^{t-\lambda} \int_{\delta}^{\infty} \alpha \theta(\psi(t)-\psi(s))^{\alpha-1} \phi_{\alpha}(\theta) S\left((\psi(t)-\psi(s))^{\alpha} \theta\right) f(s, x(s-r(s))) \psi^{\prime}(s) d s \\
& -\int_{0}^{t-\lambda} \int_{\delta}^{\infty} \alpha \theta(\psi(t)-\psi(s))^{\alpha-1} \phi_{\alpha}(\theta) S\left((\psi(t)-\psi(s))^{\alpha} \theta\right) \sigma(s) \psi^{\prime}(s) d B_{Q}^{H}(s) \|^{2} \\
& \leq 4 \alpha^{2} E \| \int_{0}^{t} \int_{0}^{\delta} \theta(\psi(t)-\psi(s))^{\alpha-1} \phi_{\alpha}(\theta) S\left((\psi(t)-\psi(s))^{\alpha} \theta\right) \\
& \times f(s, x(s-r(s))) \psi^{\prime}(s) d s \|^{2} \\
& +4 \alpha^{2} E\left\|\int_{0}^{t} \int_{0}^{\delta} \theta(\psi(t)-\psi(s))^{\alpha-1} \phi_{\alpha}(\theta) S\left((\psi(t)-\psi(s))^{\alpha} \theta\right) \sigma(s) \psi^{\prime}(s) d B_{Q}^{H}(s)\right\|^{2} \\
& +4 \alpha^{2} E \| \int_{t-\lambda}^{t} \int_{\delta}^{\infty} \theta(\psi(t)-\psi(s))^{\alpha-1} \phi_{\alpha}(\theta) S\left((\psi(t)-\psi(s))^{\alpha} \theta\right) \\
& \times f(s, x(s-r(s))) \psi^{\prime}(s) d s \|^{2} \\
& +4 \alpha^{2} E\left\|\int_{t-\lambda}^{t} \int_{\delta}^{\infty} \theta(\psi(t)-\psi(s))^{\alpha-1} \phi_{\alpha}(\theta) S\left((\psi(t)-\psi(s))^{\alpha} \theta\right) \sigma(s) \psi^{\prime}(s) d B_{Q}^{H}(s)\right\|^{2} \\
& \leq 4 \alpha^{2} M^{2} \psi^{\prime}(b) \frac{(\psi(b)-\psi(0))^{2 \alpha-1}}{2 \alpha-1} L\left(1+\mu\left(\|x\|_{\mathcal{C}}^{2}\right)\right)\left(\int_{0}^{\delta} \theta \phi_{\alpha}(\theta) d \theta\right)^{2} \\
& +4 \alpha^{2} M^{2} \psi^{\prime}(b) \frac{(\psi(b)-\psi(0))^{2 \alpha-1}}{2 \alpha-1} \sup _{t \in J}\|\sigma(s)\|_{\mathcal{L}_{2}^{0}}^{2}\left(\int_{0}^{\delta} \theta \phi_{\alpha}(\theta) d \theta\right)^{2} \\
& +4 \alpha^{2} M^{2} \frac{1}{\Gamma^{2}(\alpha+1)} \psi^{\prime}(b) \frac{(\psi(t)-\psi(t-\lambda))^{2 \alpha-1}}{2 \alpha-1} L\left(1+\mu\left(\|x\|_{\mathcal{C}}^{2}\right)\right) \\
& +4 \alpha^{2} M^{2} \frac{1}{\Gamma^{2}(\alpha+1)} \psi^{\prime}(b) \frac{(\psi(t)-\psi(t-\lambda))^{2 \alpha-1}}{2 \alpha-1} \sup _{t \in J}\|\sigma(s)\|_{\mathcal{L}_{2}^{0}}^{2},
\end{aligned}
$$

where we have used the equality

$$
\int_{0}^{\infty} \theta^{\xi} \phi_{\alpha}(\theta) d \theta=\int_{0}^{\infty} \frac{1}{\theta^{\alpha \xi}} \psi_{\alpha}(\theta) d \theta=\frac{\Gamma(1+\xi)}{\Gamma(1+\alpha \xi)}, \quad \xi \in[0,1]
$$


The right-hand side of the above inequality tends to 0 as $\lambda, \delta \rightarrow 0$. So we can obtain $E\left\|(\Phi x)(t)-\left(\Phi^{\lambda, \delta} x\right)(t)\right\|^{2} \rightarrow 0$ as $\lambda, \delta \rightarrow 0^{+}$. Since there are relatively compact sets arbitrarily close to the set $V(t)=\left\{(\Phi x)(t), x \in B_{r}\right\}$, we consequently derive that $V(t)=\left\{(\Phi x)(t), x \in B_{r}\right\}$ is also a relatively compact set in $X$.

From Claims 1-3 and the Arzola-Ascoli theorem, we deduce that $\Phi$ is a completely continuous map, then Schauder's fixed point theorem enables us to claim that the operator equation $\Phi x=x$ has at least one fixed point on $B_{r}$ which is just a mild solution for system (1.2). The proof is complete.

Compared with Theorem 4.1 in [26], the condition imposed on $f$ is easier to be satisfied in this theorem.

To establish the existence results when the associated $C_{0}$-semigroup is not necessary compact, we first require the following assumptions where $B_{r}$ is still defined as in Theorem 3.1.

$\left(\mathrm{H}_{0}^{\prime}\right) S(t)$ is continuous in the uniform operator topology for $t \geq 0$, and $\{S(t)\}_{t \geq 0}$ is uniformly bounded, i.e., there exists $M>1$ such that $\sup _{t \in[0,+\infty)}|S(t)|<M$;

$\left(\mathrm{H}_{4}\right)$ There exists a positive function $L_{f} \in L_{1}\left(J, \mathbb{R}^{+}\right)$such that, for $\forall x, y \in \mathcal{C}$,

$$
E\|f(t, x(t-r(t)))-f(t, y(t-r(t)))\|^{2} \leq L_{f}(t) E\|x(t-r(t))-y(t-r(t))\|^{2}, \quad \forall t \in J .
$$

Next, we present our second existence uniqueness result for system (1.2) based on the Banach contraction principle in the case that semigroup $\{S(t)\}_{t>0}$ is not necessary compact.

Theorem 3.2 Suppose that hypotheses $\left(H_{0}^{\prime}\right),\left(H_{1}\right)-\left(H_{4}\right)$ hold, then system (1.2) has a unique mild solution on $B_{r}$ provided that $M_{1}\left\|L_{f}(t)\right\|_{L^{1}\left(J, \mathbb{R}^{+}\right)}<1$.

Proof We omit the proof here since it can be easily verified.

Remark 3.1 The function $\sigma$ is independent of $x(t), t \in(-\tau, b]$. From the functional point of view, we have $\alpha\left(\int_{0}^{T}(\psi(t)-\psi(s))^{\alpha-1} T_{\alpha}^{\psi}(t-s) \sigma(s) \psi^{\prime}(s) d B_{Q}^{H}(s)\right)=0$.

To give our last existence results, we require the following assumptions where $\Phi, B_{r}$ are still defined as in Theorem 3.1.

$\left(\mathrm{H}_{5}\right)$ There exists a positive function $m_{f} \in L_{1}\left(J, \mathbb{R}^{+}\right)$such that, for any bounded closed subset $D \in B_{r}$, such that $\alpha\left(f(t, D(t)) \leq m_{f}(t) \alpha(D(t))\right.$;

$\left(\mathrm{H}_{6}\right)$

$$
\frac{2 M}{\Gamma(\alpha+1)}(\psi(b)-\psi(0))^{\alpha}\left\|m_{f}(t)\right\|_{L^{1}\left(J, \mathbb{R}^{+}\right)}<1
$$

To end this section, we shall present our last existence uniqueness theorem for system (1.2).

Theorem 3.3 Suppose that hypotheses $\left(H_{0}^{\prime}\right),\left(H_{1}\right)-\left(H_{3}\right),\left(H_{5}\right),\left(H_{6}\right)$ hold, then system (1.2) has at least one mild solution on $B_{r}$. 
Proof Set

$$
(\Phi x)(t):=\sum_{i=1}^{3}\left(\Phi_{i} x_{n}\right)(t),
$$

where

$$
\begin{aligned}
& \left(\Phi_{1} x_{n}\right)(t)=S_{\alpha}^{\psi}(t, 0) \phi_{n}(0) \\
& \left(\Phi_{2} x_{n}\right)(t)=\int_{0}^{t}(\psi(t)-\psi(s))^{\alpha-1} T_{\alpha}^{\psi}(t, s) f\left(s, x_{n}(s-r(s))\right) \psi^{\prime}(s) d s \\
& \left(\Phi_{3} x_{n}\right)(t)=\int_{0}^{t}(\psi(t)-\psi(s))^{\alpha-1} T_{\alpha}^{\psi}(t, s) \sigma(s) \psi^{\prime}(s) d B_{Q}^{H}(s) .
\end{aligned}
$$

In what follows, we will prove that $\Phi: B_{r} \rightarrow B_{r}$ is a condensing operator.

For any $D \subset B_{r}$, by Lemma 2.7, there exists a countable set $D_{0}=\left\{u_{n}\right\} \subset D$ such that

$$
\alpha_{C}(\Phi(D)) \leq 2 \alpha_{C}\left(\Phi\left(D_{0}\right)\right)
$$

Since $\Phi\left(D_{0}\right) \subset \Phi\left(B_{r}\right)$ is equicontinuous, we get from Lemma 2.4 that $\alpha_{C}\left(\Phi\left(D_{0}\right)\right)=$ $\max _{t \in J} \alpha\left(\Phi\left(D_{0}\right)(t)\right)$.

Obviously, we can derive

$$
0 \leq \alpha\left(\Phi_{1}\left(D_{0}\right)(t)\right)=\alpha\left(S_{\alpha}^{\psi}(t, 0) \phi(0)\right)=0
$$

Thus $\alpha\left(\Phi_{1} x_{n}\right)(t)=0$.

By Lemma 2.6 and $\left(\mathrm{H}_{5}\right)$, we have

$$
\begin{aligned}
\alpha\left(\Phi_{2}\left(D_{0}\right)(t)\right) & =\alpha\left(\int_{0}^{t}(\psi(t)-\psi(s))^{\alpha-1} T_{\alpha}^{\psi}(t, s) f\left(s, x_{n}(s-r(s))\right) \psi^{\prime}(s) d s\right) \\
& \leq \frac{2 M}{\Gamma(\alpha)} \int_{0}^{t}(\psi(t)-\psi(s))^{\alpha-1} \psi^{\prime}(s) \alpha\left(f\left(s, x_{n}(s-r(s))\right) d s\right. \\
& \leq \frac{2 M}{\Gamma(\alpha)} \int_{0}^{t}(\psi(t)-\psi(s))^{\alpha-1} \psi^{\prime}(s) m_{f}(t) \alpha(D(t)) d s \\
& \leq \frac{2 M}{\Gamma(\alpha+1)}(\psi(t)-\psi(s))^{\alpha}\left\|m_{f}(t)\right\|_{L^{1}\left(J, \mathbb{R}^{+}\right)} \alpha(D(t)) \\
& \leq \frac{2 M}{\Gamma(\alpha+1)}(\psi(b)-\psi(0))^{\alpha}\left\|m_{f}(t)\right\|_{L^{1}\left(J, \mathbb{R}^{+}\right)} \alpha(D(t)) .
\end{aligned}
$$

By Remark 3.1, we have $\alpha\left(\Phi_{3}\left(D_{0}\right)(t)\right)=0$. Thus, by Lemma 2.3 and the above inequalities, we have

$$
\begin{aligned}
& \alpha\left(\Phi\left(D_{0}\right)(t)\right) \\
& \quad \leq \alpha\left(\Phi_{1}\left(D_{0}\right)(t)\right)+\alpha\left(\Phi_{2}\left(D_{0}\right)(t)\right)+\alpha\left(\Phi_{3}\left(D_{0}\right)(t)\right) \\
& \quad \leq \frac{2 M}{\Gamma(\alpha+1)}(\psi(b)-\psi(0))^{\alpha}\left\|m_{f}(t)\right\|_{L^{1}\left(J, \mathbb{R}^{+}\right)} \alpha(D(t)) .
\end{aligned}
$$


Hence, from (3.1), (3.2), and assumption $\left(\mathrm{H}_{6}\right)$, we deduce that

$$
\begin{aligned}
\alpha(\Phi(D)(t)) & <\frac{2 M}{\Gamma(\alpha+1)}(\psi(b)-\psi(0))^{\alpha}\left\|m_{f}(t)\right\|_{L^{1}\left(J, \mathbb{R}^{+}\right)} \alpha(D(t)) \\
& <\alpha(D(t)) .
\end{aligned}
$$

Thus, $\Phi: B_{r} \rightarrow B_{r}$ is a condensing operator. It follows from Lemma 2.7 that $\Phi$ has at least one fixed point in $B_{r}$ which is actually a mild solution of system (1.2). This completes the proof of Theorem 3.3.

Remark 3.2 Having compared Theorem 3.1 and Theorem 3.2 with Theorem 3.3, we know that one can replace the strong restriction condition $\mathrm{H}_{0}$ with $\mathrm{H}_{0}^{\prime}$ on the semigroup $\{S(t)\}_{t \geq 0}$ by applying the noncompact measure method. This seems a totally new result in contrast with earlier works on fractional stochastic evolution equations. Furthermore, the obtained results can been applied to fractional stochastic partial differential equations of parabolic type.

Remark 3.3 The above theorems provide existence results of system (1.2) in the case $\psi(t) \in C^{2}(J, \mathbb{R})$ and $\psi^{\prime \prime}(t)>0, \psi^{\prime}(t)>0, \forall t \in J$, which is relatively restrictive. In fact, when $\psi(t) \in C^{2}(J, \mathbb{R})$ and $\psi^{\prime \prime}(t)<0, \psi^{\prime}(t)>0, \forall t \in J$, we can also establish the corresponding existence results of system (1.2) with appropriate modifications of the hypotheses.

\section{An example}

As an application of our obtained results, we consider the following $\psi$-Caputo fractional stochastic evolution equations driven by $\mathrm{fBm}$ :

$$
\left\{\begin{array}{l}
{ }_{0}^{C} D_{\psi}^{\alpha} x(t, z)=x_{z z}(t, z)+c_{1} x\left(\frac{t}{2}, z\right)+e^{-t} \frac{d B_{Q}^{H}(t)}{d t}, \quad t \in J=[0,1], z \in[0, \pi] \\
x(t, 0)=x(t, \pi)=0, \quad t \in J=[0,1] \\
x(t, z)=\phi(t, z), \quad t \in[-\tau, 0], z \in[0, \pi]
\end{array}\right.
$$

where ${ }_{0}^{C} D_{\psi}^{\alpha}$ is the $\psi$-Caputo fractional derivative of order $\frac{3}{4}, \psi(t)=e^{t}, f(t, x)=c_{1} x\left(\frac{t}{2}, z\right)$, $\sigma(t)=e^{-t}, r(t)=\frac{t}{2}$.

We choose the space $X=Y=L^{2}[0, \pi]$. Define an operator $A$ by $A v=v^{\prime \prime}$ with the domain $D(A)=\left\{v \in X: v, v^{\prime}\right.$ absolutely continuous, $\left.v^{\prime \prime} \in X, v(0)=v(\pi)=0\right\}$. Then $A$ generates a strongly continuous semigroup $\{S(t)\}_{t \geq 0}$ which is compact, analytic, and self-adjoint. With the above choices of $A, f, \sigma$, system (4.1) can be rewritten into the abstract form of system (1.2).

Furthermore, $A$ has a discrete spectrum, the eigenvalues are $-n^{2}, n \in N$, and the corresponding orthogonal eigenvectors are given by $e_{n}(z)=\sqrt{\frac{2}{\pi}} \sin (n z)$. Then $A z=$ $\sum_{n=1}^{\infty} n^{2}\left\langle z, e_{n}\right\rangle e_{n}$. In addition, we know that for each $v \in X, S(t) v=\sum_{n=1}^{\infty} e^{-n^{2} t}\left\langle v, e_{n}\right\rangle e_{n}$, in particular, $S(\cdot)$ is a uniformly stable semigroup and $\|S(t)\| \leq e^{-t} \leq 1:=M$.

Assume $B_{r}=\left\{x \mid x \in X, E\|x\|^{2} \leq r\right\}$, then for $\forall t \in[0,1], x \in B_{r}$, we have

$$
E\|f(t, x)\|^{2} \leq c_{1}^{2} E\left\|x\left(\frac{t}{2}, z\right)\right\|^{2} \leq 1+c_{1}^{2} E\left\|x\left(\frac{t}{2}, z\right)\right\|^{2} \leq 1+c_{1}^{2} r:=L(1+\mu(r))
$$




$$
\begin{aligned}
& \liminf _{r \rightarrow \infty} \frac{\mu(r)}{r}=\Lambda:=c_{1}^{2}, \quad \sup _{t \in J}\|\sigma(s)\|_{\mathcal{L}_{2}^{0}}^{2}=\sup _{t \in J} e^{-t} \leq 1 ; \\
& \left.M_{1}=\frac{M^{2}}{\Gamma^{2}(\alpha)} \frac{(\psi(1)-\psi(0))^{2 \alpha-1}}{2 \alpha-1} \psi^{\prime}(1)=\frac{2}{\Gamma^{2}\left(\frac{3}{4}\right)}(e-1)\right)^{\frac{1}{2}} e .
\end{aligned}
$$

Therefore, $\left(\mathrm{H}_{0}\right)-\left(\mathrm{H}_{3}\right)$ are satisfied with $M=1, L=1, \mu(r)=c_{1}^{2} r, \Lambda=c_{1}^{2}, M_{1}=\frac{2}{\Gamma^{2}\left(\frac{3}{4}\right)}(e-$ 1) $)^{\frac{1}{2}} e$. On the other hand, we can choose arbitrary constant $c_{1}$ such that $c_{1}<\left(\frac{1}{\left.\frac{6}{\Gamma^{2}\left(\frac{3}{4}\right)}(e-1)\right)^{\frac{1}{2}} e}\right)^{\frac{1}{2}}$, then we have $3 M_{1} L \Lambda<1$, this implies that all assumptions of Theorem 3.1 are satisfied. Hence, from Theorem 3.1, we can claim that system (4.1) admits at least one mild solution on $[0,1]$.

\section{Conclusion}

The aim of this manuscript was to achieve sufficient conditions to ensure the existence and uniqueness of mild solutions for a class of $\psi$-Caputo fractional stochastic evolution equations with varying-time delay driven by $\mathrm{fBm}$ using the fixed point technique, noncompact measure method, and stochastic analysis when the associated $C_{0}$-semigroup is compact or not. The obtained results generalized the classical Caputo fractional derivative case. Also, we provided an example to illustrate our results. In addition, one interesting question is to study simultaneous finite dimensional exact and approximate controllability (finite-approximate controllability) of $\psi$-Caputo fractional stochastic differential inclusions driven by $\mathrm{fBm}$ or other stochastic noise which will be treated in the future.

\section{Acknowledgements}

The author would like to thank the anonymous reviewers for their valuable comments and suggestions.

\section{Funding}

This work is supported by the National Natural Science Foundation of China (Grant No. 12001393), Natural Science Foundation of Shanxi (201901D211103).

Availability of data and materials

Not applicable.

\section{Declarations}

Competing interests

The author declares that they have no competing interests.

Authors' contributions

The author read and approved the final manuscript.

\section{Publisher's Note}

Springer Nature remains neutral with regard to jurisdictional claims in published maps and institutional affiliations.

Received: 1 March 2021 Accepted: 1 October 2021 Published online: 18 October 2021

\section{References}

1. Agrawal, O.P.: Some generalized fractional calculus operators and their applications in integral equations. Fract. Calc. Appl. Anal. 15(4), 700-711 (2017)

2. Almeida, R.: A Caputo fractional derivative of a function with respect to another function. Commun. Nonlinear Sci. Numer. Simul. 44(1), 460-481 (2017)

3. Byszewski, L., Lakshmikantham, V.: Theorem about the existence and uniqueness of a solution of a nonlocal abstract Cauchy problem in a Banach space. Appl. Anal. 40(1), 11-19 (1991)

4. Chang, S.J., Choi, J.G.: Parts formulas involving the Fourier-Feynman transform associated with Gaussian paths on Wiener space. Banach J. Math. Anal. 14(2), 503-523 (2020)

5. Chen, G., Gaans, O., Lunel, S.: Existence and exponential stability of a class of impulsive neutral stochastic partial differential equations with delays and Poisson jumps. Stat. Probab. Lett. 141(1), 7-18 (2018) 
6. Chen, P.Y., Li, Y.X., Zhang, X.P.: Cauchy problem for stochastic non-autonomous evolution equations governed by noncompact evolution families. Discrete Contin. Dyn. Syst., Ser. B 26(3), 1531-1547 (2021)

7. Chen, P.Y., Zhang, X.P.: Non-autonomous stochastic evolution equations of parabolic type with nonlocal initial conditions. Discrete Contin. Dyn. Syst., Ser. B 26(9), 4681-4695 (2021)

8. Chen, P.Y., Zhang, X.P., Li, Y.X.: Study on fractional non-autonomous evolution equations with delay. Comput. Math. Appl. 73(5), 794-803 (2017)

9. Chen, P.Y., Zhang, X.P., Li, Y.X.: A blowup alternative result for fractional nonautonomous evolution equation of Volterra type. Commun. Pure Appl. Anal. 17(5), 1975-1992 (2018)

10. Chen, P.Y., Zhang, X.P., Li, Y.X.: Existence and approximate controllability of fractional evolution equations with nonlocal conditions via resolvent operator. Fract. Calc. Appl. Anal. 23(1), 268-291 (2020)

11. Chen, P.Y., Zhang, X.P., Li, Y.X.: Approximate controllability of non-autonomous evolution system with nonlocal conditions. J. Dyn. Control Syst. 26(1), 1-16 (2020)

12. Chen, P.Y., Zhang, X.P., Li, Y.X.: Cauchy problem for fractional non-autonomous evolution equations. Banach J. Math. Anal. 14(2), 559-584 (2020)

13. Deng, S.F., Shu, X.B., Mao, J.Z.: Existence and exponential stability for impulsive neutral stochastic functional differential equations driven by fBm with noncompact semigroup via Mönch fixed point. J. Math. Anal. Appl. 467(1), 398-420 (2018)

14. Gao, D.D., Li, J.L.: Existence and mean-square exponential stability of mild solutions for impulsive stochastic partial differential equations with noncompact semigroup. J. Math. Anal. Appl. 484(1), 1-16 (2020)

15. Gao, D.D., Li, J.L.: Existence results for impulsive delayed neutral stochastic functional differential equations with noncompact semigroup. Stochastics. https://doi.org/10.1080/17442508.2021.1873340

16. Hilfer, R.: Applications of Fractional Calculus in Physics. World Scientific, Singapore (2000)

17. Jarad, F., Abdeljawad, T.: Generalized fractional derivatives and Laplace transform. Discrete Contin. Dyn. Syst., Ser. S 13(3), 709-722 (2019)

18. Kiryakova, V:: Generalized Fractional Calculus and Applications. Wiley, New York (1994)

19. Lakhel, E., McKibben, M.A.: Existence of solutions for fractional neutral functional differential equations driven by fBm with infinite delay. Stochastics 90(3), 313-329 (2018)

20. Li, K.X.: Stochastic delay fractional evolution equations driven by fractional Brownian motion. Math. Methods Appl. Sci. 38(8), 1582-1591 (2015)

21. Li, Y.J., Wang, Y.J.: The existence and asymptotic behavior of solutions to fractional stochastic evolution equations with infinite delay. J. Differ. Equ. 266(6), 3514-3558 (2019)

22. Liang, T.T., Wang, Y.J.: Sub-critical and critical stochastic quasi-geostrophic equations with infinite delay. Discrete Contin. Dyn. Syst., Ser. B 26(9), 4697-4726 (2021)

23. Nguyen, T.D.: Neutral stochastic differential equations driven by a fractional Brownian motion with impulsive effects and varying-time delays. J. Korean Stat. Soc. 43(4), 599-608 (2014)

24. Podlubny, l.: Fractional Differential Equations. Academic Press, San Diego (1999)

25. Samko, S.G., Kilbas, A.A., Marichev, O.I.: Fractional Integrals and Derivatives: Theory and Applications. Gordon \& Breach, Longhorne (1993)

26. Suechoei, A., Ngiamsunthorn, P.S.: Existence uniqueness and stability of mild solutions for semilinear $\psi$-Caputo fractional evolution equations. Adv. Differ. Equ. 114(1), 1-28 (2020)

27. Wang, R.N., Chen, D.H., Xiao, T.J.: Abstract fractional Cauchy problems with almost sectorial operators. J. Differ. Equ 252(1), 202-235 (2012)

28. Yan, Z.M., Jia, X.M.: Optimal solutions of fractional nonlinear impulsive neutral stochastic functional integro-differential equations. Numer. Funct. Anal. Optim. 40(14), 1593-1643 (2019)

29. Yang, M., Alsaedi, A., Ahmad, B., Zhou, Y.: Attractivity for Hilfer fractional stochastic evolution equations. Adv. Differ. Equ. 130(1), 1-22 (2020)

30. Yang, M., Gu, H.B.: Riemann-Liouville fractional stochastic evolution equations driven by both Wiener process and fractional Brownian motion. J. Inequal. Appl. 8(1), 1-19 (2021)

31. Yang, S., Li, Y.R.: Dynamics and invariant measures of multi-stochastic sine-Gordon lattices with random viscosity and nonlinear noise. J. Math. Phys. 62(5), 051510 (2021)

32. Zhang, X.P., Chen, P.Y., Abdelmonem, A.: Mild solution of stochastic partial differential equation with nonlocal conditions and noncompact semigroups. Math. Slovaca 69(1), 111-124 (2019)

33. Zhang, X.P., Chen, P.Y., Abdelmonem, A., Li, Y.X.: Fractional stochastic evolution equations with nonlocal initial conditions and noncompact semigroups. Stochastics 90(7), 1005-1022 (2018)

34. Zhou, Y.: Basic Theory of Fractional Differential Equations. World Scientific, Singapore (2014)

35. Zhou, Y., Jiao, F.: Existence of mild solutions for fractional neutral evolution equations. Comput. Math. Appl. 59(3), 1063-1077 (2010)

36. Zou, G.A.: Stochastic Navier-Stokes equations with Caputo derivative driven by fractional noises. J. Math. Anal. Appl. $461(1), 595-609$ (2018) 